\title{
Influence of Ultrasonic Setting on Compressive and Diametral Tensile Strengths of Glass Ionomer Cements
}

\author{
Terezinha Jesus Esteves Barata*, Eduardo Bresciani ${ }^{\mathrm{b}}$, Akimi Adachic, Ticiane Cestari Fagundes ${ }^{\mathrm{c}}$, \\ Carlos Augusto Ramos Carvalho ${ }^{\mathrm{c}, \mathrm{d}}$, Maria Fidela Lima Navarro ${ }^{\mathrm{c}}$ \\ a Department of Operative Dentistry, University of North of Paraná \\ Avenida Paris, 675, Jardim Piza 86041-100 Londrina, PR - Brazil \\ ${ }^{\mathrm{b}}$ Department of Cariology, Restorative Sciences and Endodontics, School of Dentistry, \\ University of Michigan, MI, USA \\ 'Department of Operative Dentistry, Endodontics and Dental Materials, \\ Bauru School of Dentistry, University of São Paulo - USP, Bauru - SP, Brazil \\ ${ }^{\mathrm{c}, \mathrm{d}}$ Department of Operative Dentistry, Endodontics and Dental Materials, \\ Bauru School of Dentistry, University of São Paulo - USP, Bauru - SP, Brazil \\ Department of Dental Materials and Restorative Dentistry, University of Siena, Italy
}

Received: May 22, 2007; Revised: February 4, 2008

\begin{abstract}
The aim of this study was to assess the influence of ultrasonic wave propagation on the compressive (CS) and diametral tensile (DTS) strengths of glass ionomer cements (GICs). Three variables were evaluated: conventional GICs, ultrasonic excitation and storage time (1 hour, 24 hours and 7 days). Bovine teeth molds were used for simulating a clinical ultrasonic excitation. The data were submitted to three-way ANOVA and Tukey tests $(\mathrm{P}<0.05)$. All the tested conventional GICs presented an increase in strength from 1 hour to 7 days for CS and DTS. Ultrasonic excitation resulted in a statistically significant increase in the CS, but showed no statistically significant difference in the DTS. Regardless the GICs tested the increase in strength was maturation time-dependent for all groups.
\end{abstract}

Keywords: glass ionomer cements, ultrasonic setting, compressive strength, diametral tensile strength

\section{Introduction}

The chemical bonding to tooth substrate in combination with the fluoride-releasing, low coefficient of thermal expansion and the excellent biocompatibility gives glass-ionomer cements (GICs) an important position in dentistry ${ }^{1}$. On the other hand, the acid-base setting reaction of the GICs still compromises their early strength and initial wear ${ }^{1}$. Glass ionomer goes through two stages of setting reactions ${ }^{2}$. The first stage occurs within the first 10 minutes after mixing, when the material is very susceptible to water uptake. The second stage is a slow and long-term continuation of the acid-base reaction; when it is susceptible to dehydration ${ }^{2}$. The setting reaction is a continuous process evidenced by the increase in mechanical properties of the cement with time ${ }^{3}$. Thus, the premature exposure to water leads to leaching of ions, swelling and weakening, whereas loss of water leads to shrinkage and cracking ${ }^{2}$.

Based on these findings, more recently, efforts to overcome the dilemma of the water sensitivity have been focused on the properties of setting $\mathrm{GICs}^{4}$, since the setting of the GICs can also be influenced by external factors like temperature, pressure, humidity and mixing time ${ }^{5-7}$. Studies have reported that this period can be shortened when energy, in the form of heat, is applied to the setting material ${ }^{4,7,8-12}$. The application of heat during the setting reaction of materials is not a new idea and has been described for construction engineering ${ }^{13}$. In dentistry, ultrasonic device have been successfully used removal of dental plaque and calculus on teeth surfaces ${ }^{14}$. Ultrasonic excitation can be also used as a 'command' set method by a similar process, where ultrasound is imparted from a dental scaler, ${ }^{4,-12}$. Hence, their main advantage is relative easy of handling, making them a well-liked and a widely accepted dental procedure ${ }^{14}$

Besides, the mechanical properties of the GICs can be improved by ultrasonic excitation compared to standard curing conditions, such as: hardness, compressive strength and tensile bond strength ${ }^{4,8-12}$. In addition the application of ultrasonic excitation does not modify the composition of the $\mathrm{GICs}^{4,8}$.

Despite these advantages, there is a lack of studies about the influence of externally 'command' set applications on the diametral tensile strength of glass-ionomer cements and little is known about its effects on compressive strength. In addition, other important aspect to be considered about the influence of externally 'command' set applications is that the ultrasonic waves represent a mechanical propagation of energy through a medium ${ }^{16}$. In this way, bovine teeth were prepared and used as mold material, because have an obvious relevance in the present study to simulate the clinical situation. The aim of the present study was to assess the influence of the ultrasonic excitation (UE) and standard curing conditions (SC) on the compressive (CS) and diametral tensile (DTS) strengths of conventional GICs. The null hypothesis tested was that there is no difference in the CS and DTS of conventional GICs after ultrasonic excitation.

\section{Materials and Methods}

\subsection{Bovine teeth molds preparation}

The study was approved by the Committee on Animal Care Research of the Bauru School of Dentistry. Twelve bovine mandibular molars, presenting neither enamel cracks nor fractures, were used in this study. The teeth were cleaned, stored in thymolized $0.1 \%$ saline solution at $4{ }^{\circ} \mathrm{C}$ and used within 1 month after extraction.

Each tooth was sectioned perpendicular to its longitudinal axis; $1 \mathrm{~cm}$ cervical to the occlusal pit with a water-cooled diamond saw (Extec Corp, Enfield, USA) at low-speed. This crown portion was discarded, and one disc was prepared from remaining exposed section. The disc was inspected by examination under a microscope (D.F. 
Vasconcellos, São Paulo, Brazil) at 10x magnification, to ensure that pulp had not been perforated. One disc was made from each tooth.

Six discs were prepared with $6.0 \mathrm{~mm}$ diameter $\times 4.0 \mathrm{~mm}$ height for the CS test and six discs were prepared with $6.0 \mathrm{~mm}$ diameter $\mathrm{x}$ $3.0 \mathrm{~mm}$ height for the DTS test. The discs were sectioned along the longitudinal axis in two equal hemi-sections with the same watercooled diamond saw. Each bisected disc was stored in thymolized $0.1 \%$ saline solution at $4{ }^{\circ} \mathrm{C}$ until the specimen preparation. Each bovine teeth disc was used to prepare 10 specimens.

\subsection{Samples preparation}

Six commercial restorative GICs were tested in this study. The specifications of the GICs are summarized in Table 1.

The samples for the CS and DTS tests were made by a single operator at a temperature of $23 \pm 1{ }^{\circ} \mathrm{C}$ and at a relative humidity of $50 \pm 5 \%$ in accordance with ISO specifications ${ }^{15}$.

The samples were prepared using bovine teeth mold, which were previously coated with a thin layer of petroleum jelly (Sidepal, Guarulhos, Brazil); in order to avoid the chemical bonding to tooth substrate. The weight: liquid ratios were used according to the manufacturers' instructions. The GICs were weighted in a precision balance (Mettler Toledo, Sanford, USA) and they were mixed with a plastic instrument (GC Corporation, Tokyo, Japan) on impermeable paper. After hand-mixing, the GIC was inserted into the mold, with syringe injector (Centrix, Shelton, USA).

The samples were cured randomly according to two different procedures of command set applications. Six samples of each GIC from each storage period were ultrasonically excited (UE) and six were not (SC). The protocol with this methodology was designed, as described in the current International Standard for water-based dental cements ${ }^{15}$. This Standard states that six specimens should be made for each set ${ }^{15}$.

\subsubsection{Control group: standard curing conditions (SC)}

Immediately, after the insertion of the GIC a polyester strip (Proben, Catanduva, Brazil) covered with a thin layer of petroleum jelly was placed on the material surface and the GIC was compressed using a glass plate with a pressure of $250 \mathrm{~g}$ for 2 seconds to eliminate excess GIC."

\subsubsection{Test group: ultrasonic excitation (UE)}

Following the application of hand pressure for 2 seconds, ultrasound (EMS FT-081DN Mini Piezon, Geneva, Switzerland) was applied around the mold during 20 seconds at a frequency of $25-30 \mathrm{kHz}$ (Figure 1). The ultrasonic device was used without water to cool the tip, because the water would certainly interfere with the properties of GICs. The application was performed at power position 5 on a scale of 1 to 10 using a B tip instrument. This instrument has a flat shape with $10 \mathrm{~mm}$ of length and $2 \mathrm{~mm}$ of width 12 .

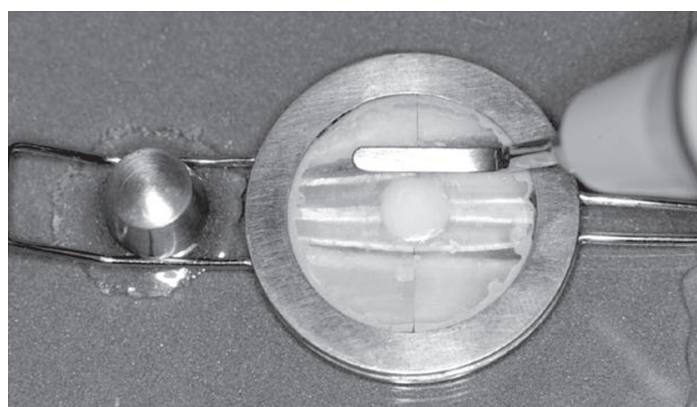

(a)

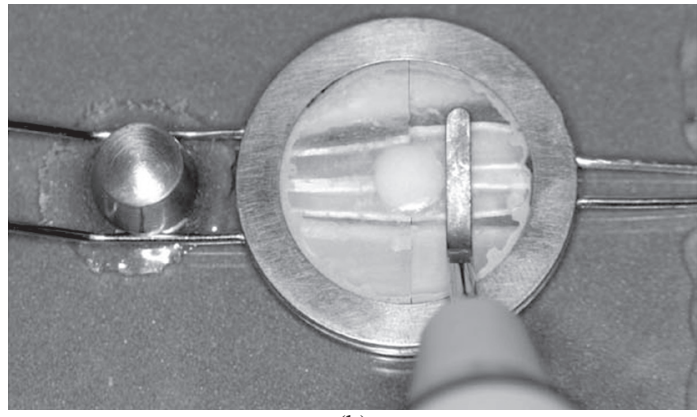

(b)

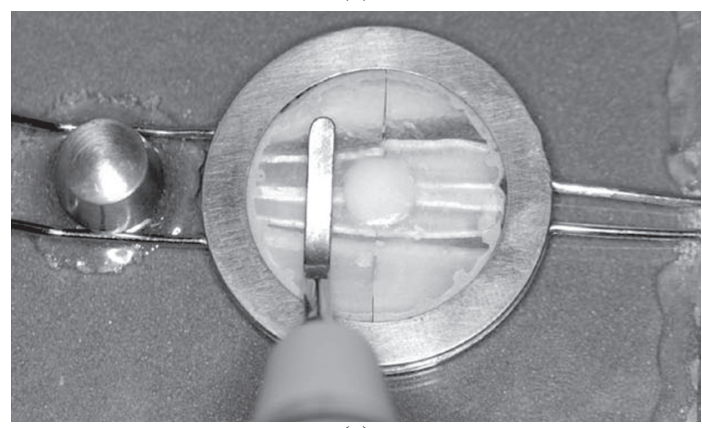

(c)

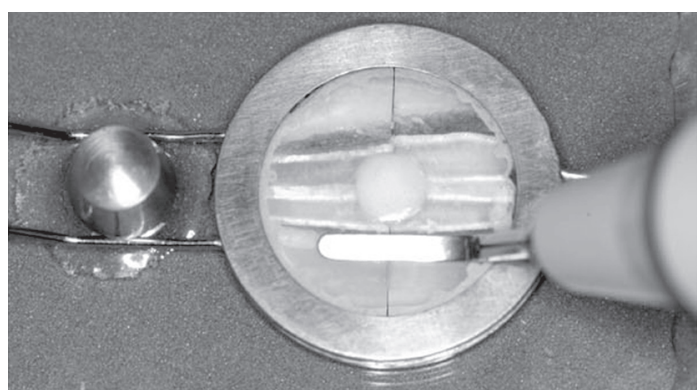

(d)

Figure 1. The ultrasonic energy was slightly applied using a B tip instrument. The application was performed around the mold for 5 seconds in each adjacent surfaces ( $\mathrm{n}=20$ seconds), no direct contact with the GIC.

Table 1. Glass ionomer cements investigated in this study.

\begin{tabular}{llcc}
\hline Glass ionomer cements* & \multicolumn{1}{c}{ Manufacturer } & Batch \# & Weight: liquid ratio \\
\hline Bioglass R & Biodinâmica, Ibiporã, Brazil & $157 / 04$ & $0.18: 0.06$ \\
Ionofill Plus & Voco, Cuxhaven, Germany & 421034 & $4.7: 1.0$ \\
Magic Glass R & Vigodent, Rio de Janeiro, Brazil & 091085 & $3.6: 1.0$ \\
Maxxion R & FGM, Joinville, Brazil & $5975-9$ & $0.17: 0.06$ \\
Vidrion R & SS White, Rio de Janeiro, Brazil & 03111039 & $1.0: 1.0$ \\
Vitro Molar & DFL, Rio de Janeiro, Brazil & 0407727 & $3.0: 1.0$ \\
\hline
\end{tabular}

*specifications: conventional restorative GIC, hand mixed and self-cured. 


\subsection{Strength measurements}

For both groups, 10 minutes after the completion of mixing, the samples were gently removed from the molds. Samples with imperfections were discarded. Afterward, the samples were immediately coated with petroleum jelly to protect the set material from the loss or gain of moisture. They were stored in $6 \mathrm{~mL}$ of deionized water in an incubator (Fanem, São Paulo, Brazil) at $37^{\circ} \pm 1{ }^{\circ} \mathrm{C}$ to simulate the oral cavity, until the tests were performed.

Ten samples for the DTS test and ten samples for the CS test were made of each GIC for each storage time: 1 hour, 24 hours and 7 days.

Tests were performed using a universal testing machine (EmicDL 5000/10000, São José dos Pinhais, Brazil) with a load cell of $100 \mathrm{~kg}$ at a crosshead speed of $1.0 \mathrm{~mm} / \mathrm{min}$ for CS and $0.5 \mathrm{~mm} / \mathrm{min}$ for the DTS test. The obtained data was converted into Kgf. $\mathrm{cm}^{-2}$.

\subsection{Statistical analysis}

All data were analyzed using the SPSS program (SigmaStat Version 13, SPSS Inc.,Chicago, USA). Since, the data were not normally distributed, suggesting a log transformation to control data heterogeneity (Log 10x).Three-way ANOVA was performed to evaluate the influence of the three variables tested: ultrasonic excitation, type of GICs and storage time. Multiple comparisons were performed using the Tukey's post hoc test when the interactions were significant. Differences were considered significant at $\mathrm{p}<0.05$

\section{Results}

All the tested GICs presented an increase in strength from 1 hour to 7 days for DTS and CS. The results of the CS and DTS measurements are summarized in Figure 2 and 3.

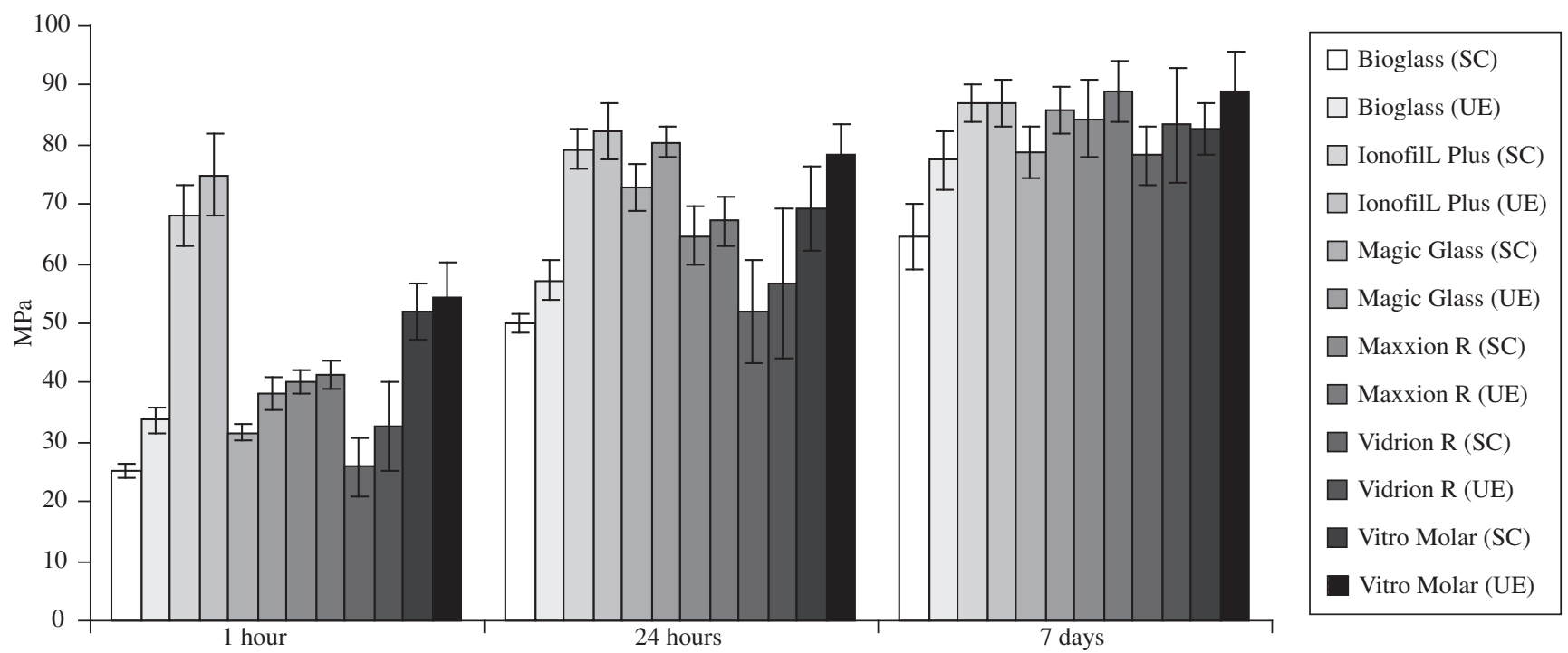

Figure 2. Mean compressive strength $(\mathrm{MPa})$ of different GICs as a function of setting method measured after 1 hour, 24 hours and 7 days $(\mathrm{n}=5)$. UE $=$ ultrasonic excitation and $\mathrm{SC}=$ standard curing. Vertical lines represent standard deviations. Ultrasonic excitation $(\mathrm{p}=0.00002), \mathrm{GICs}(\mathrm{p}=0.0000)$ and the storage time $(\mathrm{p}=0.0000)$.

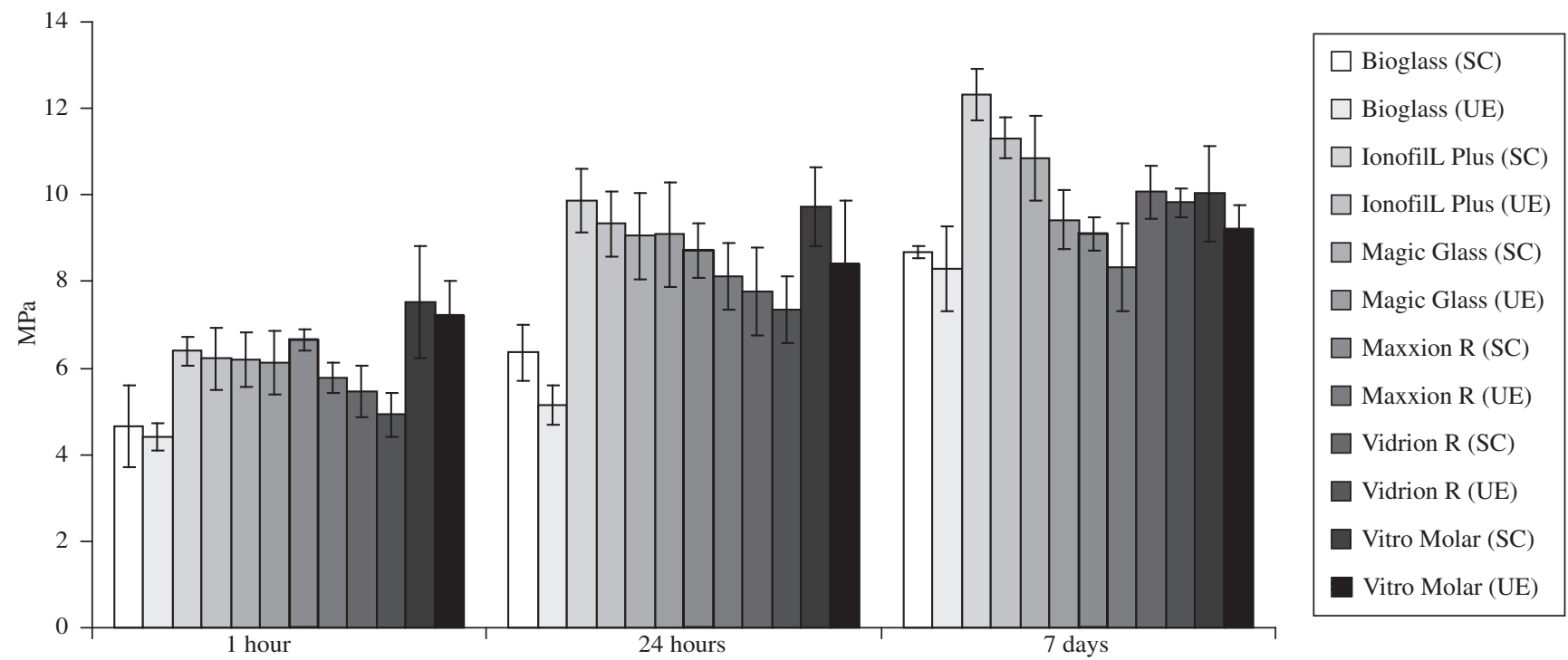

Figure 3. Mean diametral tensile (MPa) of different GICs as a function of setting method measured after 1 hour, 24 hours and 7 days $(n=5)$. UE $=$ ultrasonic excitation and SC $=$ standard curing. Vertical lines represent standard deviations. Ultrasonic excitation $(p=0.47)$, GICs $(p=0.0000)$ and the storage time $(p=0.0000)$. 


\subsection{Compressive strength}

The results showed that the 3 variables tested [ultrasonic excitation $(\mathrm{p}=0.00002)$, GICs $(\mathrm{p}=0.0000)$ and the storage time $(p=0.0000)]$ revealed statistically significant differences.

\subsection{Diametral tensile strength}

The results showed that the types of GICs $(p=0.0000)$ and the storage time $(p=0.0000)$ ] revealed statistically significant differences, while no statistically significant difference in relation to ultrasonic excitation $(\mathrm{p}=0.47)$ was observed.

\section{Discussion}

The methodology carried out in this study might be explained by the fact that the ultrasonic waves represent a mechanical propagation of energy through a medium ${ }^{16}$. It has been recognized by finite element analysis that if the mold was built with teflon (poly-tetra-fluorethylene), enamel, dentin, pulp, gold and amalgam the ultrasonic wave propagation would be 1518, 3100, 1900, 1570, 3240 and $2260 \mathrm{~m} / \mathrm{s}$ respectively ${ }^{17}$. Based on these factors it is reasonable to speculate that if the velocity is dependent on the transmission properties of the medium; the bovine teeth mold might be used to simulate the clinical condition. Similar assumption was made by Fagundes et al. ${ }^{12}$. In this context, bovine teeth, prepared and used as mold material, have an obvious relevance in our study, but some aspects should be viewed with caution:

- Cutting the bovine teeth perpendicular to its longitudinal axis due to the number and diameter of bovine tooth's root canals.

- Keeping the molds in deionized or distilled water to avoid its desiccation.

- Coating the molds with a thin layer of petroleum jelly; in order to avoid the chemical bonding of the GIC to tooth substrate.

- Making 10 samples by mold. From the preliminary laboratory studies was observed that the bovine teeth mold should be used only 10 times each, due to the lowest resistance than Teflon mold.

Under the conditions of our study the time spent for UE, frequency and power position of the ultrasonic device were performed on the basis of pilot study. This showed that a UE applied less than $20 \mathrm{sec}-$ onds around the mold does not offer neither advantage nor damage to GICs. But, if the UE was longer than 20 seconds, it would promote a decrease of strength. Our finding is in agreement with the result in bovine teeth mold ${ }^{12}$ and in disagreement with the performance in Teflon mold or stainless steel mold ${ }^{4,7,8,10}$. This would suggest that the material mold, size and shape of the tested samples must be investigated because this question has not been sufficiently clarified.

The findings of our study showed that regardless the GICs tested the CS and DTS values increased with time ( 1 hours $<24$ hours $<7$ days) for both groups (SC and UE). Application of ultrasound has resulted in an improvement statistically significant for the CS values. This observation is in agreement with previous studies ${ }^{8-11}$. Similar observations have been also reported for the tensile bond strength and hardness $s^{4,7,12}$. On the other hand, our results still revealed that regardless the GICs tested, the DTS was not influenced by the UE. Based on these results the null hypothesis was rejected for CS and accepted for DTS. The reason for this is not clear, but it might be associated to the different fracture modes and viscoelastic behavior of the GICs ${ }^{18-19}$. Cohesion between the materials is identical in both tests but the direction of force is reversed ${ }^{2}$. For the DTS test the samples are compressed diametrically, while for the CS test, the samples are placed in a vertical position, with force incident on the long axis ${ }^{18}$.
These results indicated that the application of the ultrasound has a significant effect on the setting and reaction time of $\mathrm{GICs}^{4,7,8}$, since a significant temperature rise in the GICs can be obtained with ultrasonic excitation ${ }^{4,7,8}$. The mechanism of the accelerated setting and increased mechanical properties is not yet clear, but preliminary studies ${ }^{4,7-11}$ suggested that adding kinetic energy from the ultrasonic device to the material can enhance the rate of the reaction conversion due to the increase in temperature. Beside the generation of heat, ultrasound may also contribute to acceleration of the reaction by de-clustering glass particles and enhancing the diffusion of the reaction components. Thus the GICs can be condensed by a reduction of porosities or in a closer packing of the particles ${ }^{4,7-11}$.

It is important to state that the findings of this study must be interpreted carefully, considering the limited nature of the in vitro test used, which does not necessarily replicate clinical situation. Nevertheless, the results suggest that the UE compared with the SC could have clinical implications in GICs. A comprehensive scientific understanding of the relationships between ultrasonic treatment and physical properties of GICs is necessary. In addition, an understanding of the clinical effects of ultrasonic treatment on the dental GICs and the surrounding tooth material is essential for effective clinical application.

\section{Conclusion}

The increase in strength was maturation time-dependent for all groups. Ultrasound excitation increased the compressive strength, but had no influence on the diametral tensile strength.

\section{Acknowledgments}

TJE Barata was supported by a fellowship from Coordination of Training of Higher Education Graduate (CAPES), Brazil.

\section{References}

1. Tyas MJ. Milestones in adhesion: glass ionomer cements. J Adhes Dent. 2003; 5(4): 259-266

2. Darvell BW. Materials science for dentistry. $6^{\text {th }}$ ed. Hong Kong, B.W. Darvell; 2000. p. 18-20.

3. Mitra SB, Kedrowski BL. Long-term mechanical properties of glass ionomers. Dent Mater. 1994; 10(2): 78-82.

4. Towler MR, Bushby AJ, Billington RW, Hill RG. A preliminary comparison of the mechanical properties of chemically cured and ultrasonically cured glass ionomer cements, using nano-indentation techniques. Biomaterials. 2001; 22(11): 1401-1406.

5. Prentice LH, Tyas MJ, Burrow MF. The effect of mixing time on the handling and compressive strength of an encapsulated glass-ionomer cement. Dent Mater. 2005; 21(8): 704-708.

6. van Duinen RN, Kleverlaan CJ, de Gee AJ, Werner A, Feilzer AJ. Early and long-term wear of 'fast-set' conventional glass-ionomer cements. Dent Mater. 2005; 21(8): 716-720.

7. Algera TJ, Kleverlaan CJ, Prahl-Andersen B, Feilzer AJ. The influence of environmental conditions on the material properties of setting glassionomer cements. Dent Mater. 2006; 22(9): 852-856.

8. Algera TJ, Kleverlaan CJ, de Gee AJ, Prahl-Andersen B, Feilzer AJ. The influence of accelerating the setting rate by ultrasound or heat on the bond strength of glass ionomers used as orthodontic bracket cements. Eur $J$ Orthod. 2005; 27(5): 472-476.

9. Kleverlaan CJ, van Duinen RN, Feilzer AJ. Mechanical properties of glass ionomer cements affected by curing methods. Dent Mater. 2004; 20(1): 45-50.

10. Twomey E, Towler MR, Crowley CM, Doyle J, Hampshire S. Investigation into the ultrasonic setting of glass ionomer cements. Parte II Setting times and compressive strengths. J Mater Sci, 2004; 39(14): 4631-4632. 
11. Tanner DA, Rushe N, Towler MR. Ultrasonically set glass polyalkenoate cements for orthodontic applications. J Mater Sci Mater Med. 2006; 17(4): 313-318.

12. Fagundes TC, Barata TJ, Bresciani E, Cefaly DF, Carvalho CA, Navarro MF. Influence of ultrasonic setting on tensile bond strength of glassionomer cements to dentin. J Adhes Dent. 2006; 8(6): 401-407.

13. Chotard T, Gimet-Breart N, Smith A, Fargeot D, Bonnet JP, Gault C. Application of ultrasonic testing to describe the hydration of calcium aluminate cement at the early age. Cement and Concrete Research, 2001; 31(3): 405-412.

14. Kocher T, Fanghanel J, Schwahn C, Ruhling A. A new ultrasonic device in maintenance therapy: perception of pain and clinical efficacy. $J$ Clin Periodontol. 2005; 32(4): 425-429.
15. International Standard IS0 9917: Dental water-based cements, 1991.

16. Laird WR, Walmsley AD. Ultrasound in dentistry. Part 1 - biophysical interactions. J Dent. 1991;19(1): 14-17.

17. Ghorayeb SR, Xue T, Lord W. A finite element study of ultrasonic wave propagation in a tooth phantom. J Dent Res. 1998; 77(1): 39-49.

18. Yap AU, Pek YS, Cheang P. Physico-mechanical properties of a fast-set highly viscous GIC restorative. J Oral Rehabil. 2003; 30(1): 1-8.

19. Yamazaki T, Schricker SR, Brantley WA, Culbertson BM, Johnston W. Viscoelastic behavior and fracture toughness of six glass-ionomer cements. J Prosthet Dent. 2006; 96(4): 266-272. 
